\title{
Accurate strain measurements in highly strained Ge microbridges
}

\author{
A. Gassenq, ${ }^{1,2}$ S. Tardif, ${ }^{1,2}$ K. Guilloy, ${ }^{1,2}$ G. Osvaldo Dias, ${ }^{1,3}$ N. Pauc, ${ }^{1,2}$ I. Duchemin,,${ }^{1,2}$ \\ D. Rouchon, ${ }^{1,3}$ J.-M. Hartmann, ${ }^{1,3} \mathrm{~J}$. Widiez, ${ }^{1,3} \mathrm{~J}$. Escalante, ${ }^{1,2}$ Y.-M. Niquet, ${ }^{1,2}$ R. Geiger, ${ }^{4}$ \\ T. Zabel, ${ }^{4}$ H. Sigg, ${ }^{4}$ J. Faist, ${ }^{5}$ A. Chelnokov, ${ }^{1,3}$ F. Rieutord, ${ }^{1,2}$ V. Reboud, ${ }^{1,3}$ and V. Calvo ${ }^{1,2}$ \\ ${ }^{1}$ Univ. Grenoble Alpes, 38000 Grenoble, France \\ ${ }^{2}$ CEA-INAC, 17 rue des Martyrs, 38000 Grenoble, France \\ ${ }^{3}$ CEA-LETI, Minatec Campus, 17 rue des Martyrs, 38054 Grenoble, France \\ ${ }^{4}$ Laboratory for Micro- and Nanotechnology, Paul Scherrer Institut, 5232 Villigen, Switzerland \\ ${ }^{5}$ Institute for Quantum Electronics, ETH Zurich, 8093 Zürich, Switzerland
}

(Received 1 April 2016; accepted 31 May 2016; published online 13 June 2016)

\begin{abstract}
Ge under high strain is predicted to become a direct bandgap semiconductor. Very large deformations can be introduced using microbridge devices. However, at the microscale, strain values are commonly deduced from Raman spectroscopy using empirical linear models only established up to $\varepsilon_{100}=1.2 \%$ for uniaxial stress. In this work, we calibrate the Raman-strain relation at higher strain using synchrotron based microdiffraction. The Ge microbridges show unprecedented high tensile strain up to $4.9 \%$ corresponding to an unexpected $\Delta \omega=9.9 \mathrm{~cm}^{-1}$ Raman shift. We demonstrate experimentally and theoretically that the Raman strain relation is not linear and we provide a more accurate expression. Published by AIP Publishing. [http://dx.doi.org/10.1063/1.4953788]
\end{abstract}

Strain engineering has become a widely used strategy to enhance the performance of semiconductor devices ${ }^{1}$ such as transistors, ${ }^{2}$ modulators, ${ }^{3}$ piezoelectrics, ${ }^{4}$ and semiconductor lasers, ${ }^{5}$ to name a few. Many material properties like electronic band structures can indeed be tailored by strain. For germanium, it has been predicted that high tensile strain can tune the relative band gap energies, improving light emission and transforming it into a direct band gap material, ${ }^{6-9}$ opening the way to mid-infrared lasers fully compatible with Complementary Metal Oxide Semiconductor (CMOS) technology. The tensile strain needed to reach a direct bandgap has been theoretically estimated to be around 4.6\% (Refs. 9 and 10) for a uniaxial loading along $\langle 100\rangle$. Reaching such large strain values while retaining crystal integrity is extremely challenging. Several methods are currently being explored, ${ }^{11-14}$ and the highest strains are obtained by strain redistribution..$^{10,15}$ The attained strains exceed in a radical way the intrinsic strain limits of conventional epitaxial growth. ${ }^{16,17}$

The method of choice for a direct, model-free determination of strain in a crystalline material is X-ray diffraction. At synchrotrons, latest developments allow strain measurements down to the sub-micrometer scale. ${ }^{18-20}$ However, due to the accessibility, micro-Raman spectroscopy is routinely used in the laboratory to quantify the local strain from a measurement of the peak frequency shift of an optical phonon mode. To determine the strain, experimental strain-to-frequency conversion rules are used. The rules are specific for each material and orientation of the strain. ${ }^{21,22}$ For Germanium $(\mathrm{Ge})$, the Raman shift conversion rules were experimentally established for uniaxial strain up to $1.2 \%,{ }^{22}$ which is much lower than the theoretical ideal yield strength of monocrystalline $\mathrm{Ge}$ of approximately $18 \%{ }^{23}$ In practice, crystalline defects and roughness at the interfaces reduce the limit of rupture. Nevertheless, the strains achieved now in Ge micro-structures are several-fold higher ${ }^{10,15}$ than what was previously studied to establish the Raman-strain relation. Therefore, the Raman strain shift coefficient needs to be calibrated up to higher level of strain in order to give access to accurate high strain micromeasurements in $\mathrm{Ge}$ with laboratory equipment.

In this work, we demonstrate accurate measurements of elevated strain in Ge via Raman spectroscopy and synchrotron based X-ray micro-diffraction in order to calibrate the strain-to-Raman-shift up to tensile strain of an unprecedented level of $4.9 \%$, corresponding to $9.9 \mathrm{~cm}^{-1}$ Raman wavenumber shift.

Stress in the semiconductor layer is applied in a controllable way by strain redistribution. The microbridge geometry was initially proposed in $\mathrm{Si}^{24}$ and later in Ge. ${ }^{14}$ At that time, ${ }^{14}$ only limited strain (Raman shifts up to $4.8 \mathrm{~cm}^{-1}$ ) was reached in Ge before fracturing the microbridges, most likely due to the presence of an array of misfit dislocations at the $\mathrm{Ge} / \mathrm{Si}$ interface. For this work, we used high crystalline quality 200-mm optical Ge-on-Insulator (GeOI) wafers fabricated by Smart-Cut ${ }^{\mathrm{TM}}$ technology ${ }^{25-27}$ in order to shift the mechanical failure of microbridges to higher strain. ${ }^{15,25}$ Fig. 1(a) presents the process flow used to fabricate Ge microbridges (Fig. 1(b)). Ge layers are $0.35 \mu \mathrm{m}$ thick with a biaxial strain evaluated at $0.16 \%$. Microbridge patterning was performed using e-beam lithography and $\mathrm{Ge}$ etching in an inductively coupled plasma reactor. The Ge membranes were then released using under-etching in a dedicated etching reactor combining anhydrous $\mathrm{HF}$ vapors and alcohol vapors. Thanks to the under-etching, tensile strain was concentrated in the narrowest part of the suspended microbridge. The strain homogeneity in the central region allows measuring locally the material with $1 \mu \mathrm{m}$-diameter probes. ${ }^{28}$

As far as strain micro-characterization is concerned, Raman spectroscopy has the advantage of being relatively well spatially resolved, fast, and widely available in laboratories. Under optical excitation, lattice vibrations generate a variation in the electrical susceptibility of the Ge crystal, which gives rise to Rayleigh and Raman scattering. Mechanical stress affects the spectral frequencies of the Raman modes, giving access to the induced strain. The 
(a)

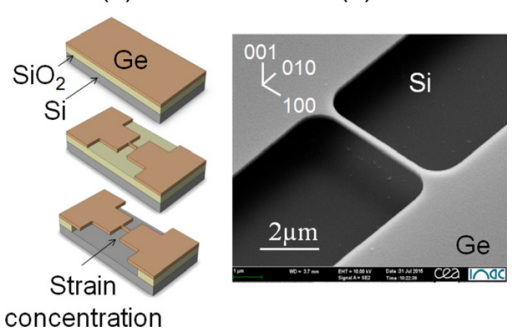

FIG. 1. (a) Process flow used for the fabrication of suspended microbridges from optical GeOI wafers. (b) Fabricated Ge microbridges with the indicated crystallographic axis.

relationship between strain and Raman wavenumber shift will be detailed hereafter. In this work, a micro-Raman spectrometer with a 532 or $785 \mathrm{~nm}$ wavelength excitation laser is used to probe the strain in Ge microbridges. The light was focused on the sample surface with a $100 \times$ short working distance objective. Since the excitation polarization can influence the intensity of the different Raman modes, ${ }^{29,30}$ we have used an unpolarized source. In our case, only one Transverse Optic (TO) retro-diffused mode is observed for an uniaxial stress along $\langle 100\rangle .{ }^{14,22}$ Therefore, the Raman peak positions and intensities were not affected by the sample orientation. Measured Raman spectra were compared for both wavelengths. Since the measured Raman spectral shifts were similar for low input power, the $785 \mathrm{~nm}$ wavelength was chosen for a deeper probing depth $(\sim 200 \mathrm{~nm}$ (Ref. 31)) with a resulting spot diameter around $1 \mu \mathrm{m}$. Power dependence measurements were performed in order to quantify and correct heating effects. ${ }^{32}$ From this calibration, the laser intensity, fixed at $9 \mu \mathrm{W}$, was focused on the sample. The heating effect was lower than the indicated $0.1 \mathrm{~cm}^{-1}$ uncertainty coming from the Raman spectrometer. The Raman spectral shift was measured by fitting the Raman spectra with Lorentzian functions. A bulk Ge (001) substrate was systematically used as a reference for $0 \%$ strain. Fig. 2 presents the measured spectra for several micro-bridges. The typical dimensions ( $\mathrm{L}, \mathrm{d}$, and e) used to tune the strain are indicated in the scale. For $\mathrm{d}=250 \mathrm{~nm}$, the spectral shift reaches $9.9 \pm 0.1 \mathrm{~cm}^{-1}$, which is the highest reported value in the literature up to date for $\mathrm{Ge}$ micro-bridges. Using the empirical model commonly employed for uniaxial stress in $\mathrm{Ge},{ }^{9,10,14,33}$ such very high Raman spectral shift should be converted into $6.4 \%$ of strain. However, the validity of the Raman linear conversion rules initially established only up to $1.2 \%$ in $\mathrm{Ge}^{22}$ has to be verified at such higher level of strain. Indeed, nonlinear Raman-strain relation has been already reported in the literature for other materials. ${ }^{34-39}$ Therefore, direct strain measurements in such objects are now absolutely needed for calibrating the Raman-strain relation at higher strain levels.

The strain state in the Ge microbridges was measured using X-ray Laue and rainbow-filtered Laue microdiffraction at beam-line BM32 of the European Synchrotron Radiation Facility in Grenoble. ${ }^{28,40}$ The energy spectrum of the white synchrotron X-ray beam ranged from 5 to $25 \mathrm{keV}$, and the beam was focused (with a $0.5 \mu \mathrm{m} \times 0.5 \mu \mathrm{m}$ spot size) on the central part of the microbridges. The Laue diffraction patterns were collected on a 2 dimensional MARCCD165

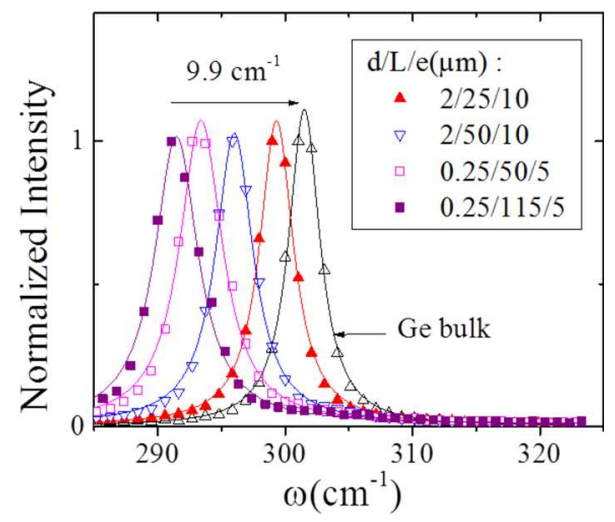

FIG. 2. Raman spectra of different Ge microbridges.

detector. For rainbow-filtered measurements, a diffracting diamond plate was inserted in the beam path to control the incident energy spectrum.

Typical Laue diffraction patterns for different microbridges on the same chip are shown in Figs. 3(a) and 3(b). Fig. 3(a) is a zoomed-in view of Fig. 3(b) showing the evolution of two diffraction spots corresponding to the Miller indices of the 337 and 224 Bragg reflections of the Si substrate and of several suspended Ge microbridges processed on the same chip. Depending on the bridge design (parameters d, L, and e indicated in the inset), a shift of the Ge spot positions is observed. The full widths at half maximum values are constant (1.3 \pm 0.1 pixels) which indicate that the strain stays homogeneous when the deformation increases. The deviatoric strain tensor was calculated from the peak positions on the Laue pattern using the software LaueTools. ${ }^{41}$ The longitudinal strain value was obtained from the deviatoric strain tensor by considering no stress normal to the free surfaces. ${ }^{28}$ Measured strains are indicated in the legend; $4.8 \%$ is achieved in this device. A maximum of $4.9 \%$ will be presented hereafter in even narrower microbridges $(250 \mathrm{~nm}$ width). All measured strain tensors correspond to an uniaxial stress configuration, i.e., tensile strain parallel to the stress direction and compressive strain perpendicular to the stress direction, the ratio between parallel and perpendicular corresponding to the Poisson ratio of Ge. ${ }^{28,42}$ Additional direct measurements of the longitudinal strain were performed on a few microbridges using the rainbow filter. ${ }^{40}$ Only few microbridges carefully chosen over the whole strain range were measured during the limited time span of the beam-time. The rainbow-filtered measurement in a $2.9 \%$ strained membrane is shown as an example in Fig. 3(d), where the intensities of 5 different Bragg reflections are plotted as a function of the angle of the diamond plate. The corresponding Bragg reflections on the detector are indicated by circles in Fig. 3(b). The intensity drops when the energy of the Bragg reflection corresponds to a diffraction of the diamond plate which allows to measure directly the lattice parameter. Knowing the energy of the Bragg reflection provides the value of the spacing of the atomic planes. ${ }^{40}$ We were thus able to locally access the strain along the $\langle 100\rangle$ direction in Ge microbridges using two micro-diffraction techniques.

We can now link such Raman spectral shifts to the strain obtained from X-ray micro-diffraction measurements performed on the same microbridges. Fig. 4 presents the 
(a) $337 \& 224$ diffracted peaks

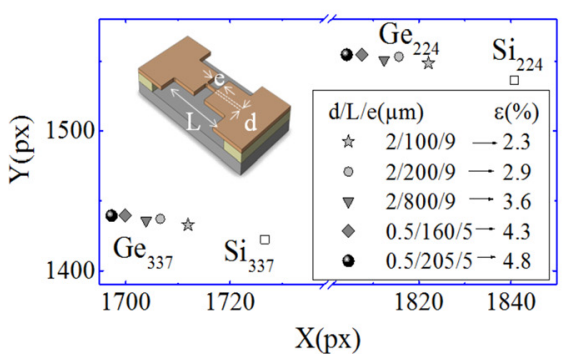

(b) Laue diffraction patterns

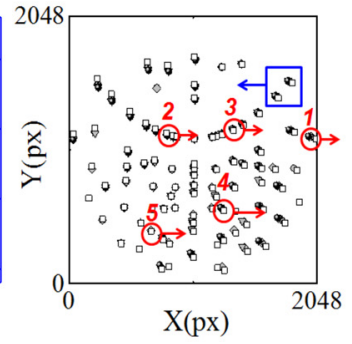

(c) Rainbow-filtered peaks

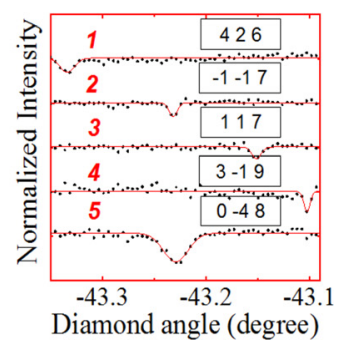

FIG. 3. (a) Zoom on the 337 and 22 4 Bragg reflections from the (b) full Laue diffraction pattern of the superimposed diffraction patterns from the $\mathrm{Si}$ substrate and several microbridges with different dimensions; (c) Intensity of 5 selected Bragg reflections as a function of the diamond plate angle in the rainbow-filtered $\mu$ Laue technique for the membrane with the $2.9 \%$ strain. measured strain as a function of the spectral Raman shift. The Raman shift is attributed to the Gamma TO retrodiffused mode resulting from to a $\langle 100\rangle$ stress, ${ }^{22}$ and the strain was measured along the $\langle 100\rangle$ crystallographic orientation. Diffraction (circles) and rainbow-filtered Laue diffraction (stars) measurements exhibit a very good agreement which confirms the absence of stress normal to the free surfaces. $^{28} \mathrm{~A}$ clearly nonlinear relationship is found, with the maximal strain evaluated at $4.9 \pm 0.1 \%$ for a shift of $9.9 \pm 0.1 \mathrm{~cm}^{-1}$.

Till now, linear relationships were widely used in the literature to link Raman shift to strain in Ge using the Raman secular equation. ${ }^{21,22,43}$ The linearity was experimentally confirmed for several situations with relatively low strain (up to $1.2 \%$ uniaxial stress in $\mathrm{Ge},{ }^{22}$ up to $2.6 \%$ bi-axial stress in $\mathrm{Ge} ; ;^{44,45}$ and up to $2.6 \%$ uniaxial stress in $\mathrm{Si}^{46}$ ). For uniaxial stress along $\langle 100\rangle$ in Ge, the commonly used linear empirical relation is given by Equation (1) with $\varepsilon$ in $\%$ and $\omega$ in $\mathrm{cm}^{-1} \cdot{ }^{14,22}$ The standard error on the strain-shift coefficient can be evaluated to approx. 0.02 coming from the phonon deformation potential uncertainty ${ }^{22}$

$$
\Delta \varepsilon_{100}=0.65( \pm 0.02) \times \Delta \omega .
$$

The deviation from linearity of the Raman shift versus strain for the $\langle 100\rangle$ loading is evident from the data shown in Fig. 4. To provide the revisited strain-shift relation over the $0 \%-5 \%$ tensile strain and $0-10 \mathrm{~cm}^{-1}$ shift range, we have fitted our experimental values by Equation (2). The indicated uncertainties are evaluated by the fit deviation

$$
\Delta \varepsilon_{100}=0.68( \pm 0.02) \times \Delta \omega-0.019( \pm 0.002) \times \Delta \omega^{2} .
$$

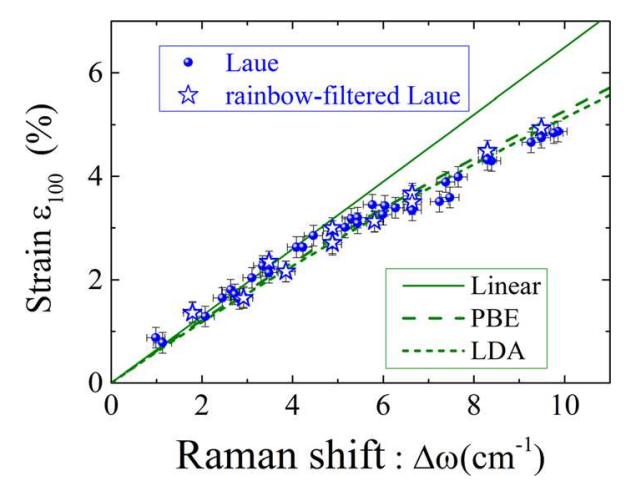

FIG. 4. Raman strain relation: measured strain by micro-XRD (blue circles and stars), simulated strain by ab initio calculations (dotted green lines), and linear empirical dependence (solid green line).
Taking into account the coefficient uncertainties, we find a good agreement between Eqs. (1) and (2) at low strain values $(<2.5 \%)$, which is expected. At higher strains, the difference between Eqs. (1) and (2) is substantial: for example, from Eq. (1) we obtain that a Raman shift of $9.9 \mathrm{~cm}^{-1}$ would be converted to $6.4 \%$ strain, while the actual strain value is $4.9 \%$, instead. To support the experimental findings, we performed $a b$-initio calculations of the Raman shifts. The dependence of the TO phonon wavenumber on strain was determined up to 5.5\% from both Local-Density Approximation (LDA) and Perdew-Burke-Ernzerhof (PBE) exchange correlation functionals using the Abinit Density Functional Theory (DFT) package. ${ }^{47-49}$ In order to circumvent metallicity problems arising from DFT calculation in crystalline Ge, we enforced a complete occupation of the valence band structure over the whole Brillouin zone, corresponding effectively to a $0 \mathrm{~K}$ electronic temperature. The relaxation of the strained crystalline structure along the axis perpendicular to the constraint has been obtained for the 8 atoms orthorhombic supercell. Response function phonon calculations were performed on the resulting relaxed 2 atoms unit cell. For TO phonons which are measured in our experiments, PBE and LDA calculations give similar results and exhibit a very good agreement to the experimental values (Fig. 4) which supports the revisited Ramanstrain relation (Eq. (2)). In addition, the Grüneisen parame$\operatorname{ter}^{34-39}$ has been extracted from our data. Knowing the lattice parameter values (measured by Rainbow filtered measurement) and the full strain tensors (measured by Laue micro-diffraction), we have compared the measured volume change to the measured Raman frequency. A Grüneisen parameter of 1.1 was found up to $2.0 \%$ in good agreement with the literature for the well-known low strain regime. ${ }^{35,38,50,51}$ However, a revisited value of 1.4 was found for the higher strain levels (up to $5.0 \%)$. Such data are in good agreement with our ab-initio calculations. Therefore, the constant Grüneisen parameter formalism cannot describe perfectly the high strain regime compared to $a b$-initio calculation. As a consequence, we provide a more accurate Raman-strain relation (Eq. (2)) compared to the commonly used linear relation (Eq. (1)) for high strain measurement in Ge micro-bridges. Reported high strain values in the pioneer works ${ }^{9,10,14}$ using the linear model (Eq. (1)) for high levels of strain are thus overestimated.

To sum up, thanks to the high crystalline quality of $200 \mathrm{~mm}$ optical GeOI wafers, large uniaxial strain in suspended Ge microbridges was achieved up to a recordbreaking 4.9\%, corresponding to a Raman spectral shift of $9.9 \mathrm{~cm}^{-1}$. A large range of strain was measured by X-ray 
micro-diffraction and the relationship between experimental Raman shifts and strain was determined. The obtained unexpected deviation from the linear dependence was confirmed by ab-initio calculations. An upgraded relationship between Raman shift and strain is provided. It allows for accurate measurements of extreme strain in Ge with Raman spectroscopy, which is a key for the realization of a direct bandgap material for CMOS compatible laser application. Our work shows also the high potential of micro-bridge processing combined to synchrotron based measurement to probe the material properties of heavily strained semiconductors.

The authors would like to thank the Platforme de Technologie Amont in Grenoble for clean room facilities and the beamline BM32 at ESRF for synchrotron based measurement. This work was supported by the CEA DRFDRT Phare projects "Photonics" and "Operando," the CEAEnhanced Eurotalent project "Straintronics," as well as the Swiss National Science foundation SNF.

${ }^{1}$ Y. Sun, S. E. Thompson, and T. Nishida, Strain Effect in Semiconductors (Springer US, 2010).

${ }^{2}$ J. A. del Alamo, Nature 479, 317 (2011).

${ }^{3}$ R. S. Jacobsen, K. N. Andersen, P. I. Borel, J. Fage-Pedersen, L. H. Frandsen, O. Hansen, M. Kristensen, A. V. Lavrinenko, G. Moulin, H. Ou, C. Peucheret, B. Zsigri, and A. Bjarklev, Nature 441, 199 (2006).

${ }^{4}$ T. Takeuchi, C. Wetzel, S. Yamaguchi, H. Sakai, I. Akasaki, Y. Kaneko, S. Nakagawa, Y. Yamaoka, and N. Yamada, Appl. Phys. Lett. 73, 1691 (1998).

${ }^{5}$ A. R. Adams, Electron. Lett. 22, 249 (1986).

${ }^{6}$ M. El Kurdi, G. Fishman, S. Sauvage, and P. Boucaud, J. Appl. Phys. 107, 013710 (2010).

${ }^{7}$ J. R. Sanchez-Perez, C. Boztug, F. Chen, F. F. Sudradjat, D. M. Paskiewicz, R. B. Jacobson, M. G. Lagally, and R. Paiella, Proc. Natl. Acad. Sci. U.S.A. 108, 18893 (2011).

${ }^{8}$ O. Aldaghri, Z. Ikonić, and R. W. Kelsall, J. Appl. Phys. 111, 053106 (2012).

${ }^{9}$ R. Geiger, T. Zabel, and H. Sigg, Front. Mater. 2, 1 (2015).

${ }^{10}$ D. S. Sukhdeo, D. Nam, J.-H. Kang, M. L. Brongersma, and K. C. Saraswat, Photonics Res. 2, A8 (2014).

${ }^{11}$ A. Ghrib, M. El Kurdi, M. Prost, S. Sauvage, X. Checoury, G. Beaudoin, M. Chaigneau, R. Ossikovsk, I. Sagnes, and P. Boucaud, Adv. Opt. Mater. 3, 353 (2015).

${ }^{12}$ C. Boztug, J. R. Sánchez-Pérez, F. F. Sudradjat, R. Jacobson, D. M. Paskiewicz, M. G. Lagally, and R. Paiella, Small 9, 622 (2013).

${ }^{13}$ M. El Kurdi, H. Bertin, E. Martincic, M. De Kersauson, G. Fishman, S. Sauvage, A. Bosseboeuf, and P. Boucaud, Appl. Phys. Lett. 96, 041909 (2010).

${ }^{14}$ M. J. Süess, R. Geiger, R. A. Minamisawa, G. Schiefler, J. Frigerio, D. Chrastina, G. Isella, R. Spolenak, J. Faist, and H. Sigg, Nat. Photonics 7, 466 (2013).

${ }^{15}$ A. Gassenq, K. Guilloy, G. Osvaldo Dias, N. Pauc, D. Rouchon, J.-M. Hartmann, J. Widiez, S. Tardif, F. Rieutord, J. Escalante, I. Duchemin, Y.M. Niquet, R. Geiger, T. Zabel, H. Sigg, J. Faist, A. Chelnokov, V. Reboud, and V. Calvo, Appl. Phys. Lett. 107, 191904 (2015).

${ }^{16}$ P. M. J. Marée, J. C. Barbour, J. F. Van Der Veen, K. L. Kavanagh, C. W. T. Bulle-Lieuwma, and M. P. A. Viegers, J. Appl. Phys. 62, 4413 (1987).

${ }^{17}$ J. W. Matthews and A. E. Blakeslee, J. Cryst. Growth 27, 118 (1974).

${ }^{18}$ K. Guilloy, N. Pauc, A. Gassenq, P. Gentile, S. Tardif, F. Rieutord, and V. Calvo, Nano Lett. 15, 2429 (2015).

${ }^{19}$ T. Etzelstorfer, M. J. Süess, G. L. Schiefler, V. L. R. Jacques, D. Carbone, D. Chrastina, G. Isella, R. Spolenak, J. Stangl, H. Sigg, and A. Diaz, J. Synchrotron Radiat. 21, 111 (2014).

${ }^{20}$ G. Capellini, G. Kozlowski, Y. Yamamoto, M. Lisker, C. Wenger, G. Niu, P. Zaumseil, B. Tillack, A. Ghrib, M. De Kersauson, M. El Kurdi, P. Boucaud, and T. Schroeder, J. Appl. Phys. 113, 013513 (2013).

${ }^{21}$ E. Anastassakis, A. Pinczuk, E. Burstein, F. H. Pollak, and M. Cardona, Solid State Commun. 88, 1053 (1993).

${ }^{22}$ F. Cerdeira, C. J. Buchenauer, F. H. Pollak, and M. Cardona, Phys. Rev. B 5, 580 (1972).
${ }^{23}$ A. L. Ruoff, "Applications and mechanical properties," in High-Pressure Science and Technology (Springer USA, 1979), Vol. 2, pp. 525-548.

${ }^{24}$ R. A. Minamisawa, M. J. Süess, R. Spolenak, J. Faist, C. David, J. Gobrecht, K. K. Bourdelle, and H. Sigg, Nat. Commun. 3, 1096 (2012).

${ }^{25}$ V. Reboud, J. Widiez, J.-M. Hartmann, G. Osvaldo Dias, D. Fowler, A. Chelnokov, A. Gassenq, K. Guilloy, N. Pauc, V. Calvo, R. Geiger, T. Zabel, J. Faist, and H. Sigg, Proc. SPIE 9367, 936714 (2015).

${ }^{26}$ E. Augendre, L. Sanchez, L. Benaissa, T. Signamarcheix, J.-M. Hartmann, C. Le Royer, M. Vinet, W. Van Den Daele, J.-F. Damlencourt, K. Romanjek, A. Pouydebasque, P. Batude, C. Tabone, F. Mazen, A. Tauzin, N. Blanc, M. Pellat, J. Dechamp, M. Zussy, P. Scheiblin, M.-A. Jaud, C. Drazek, C. Maurois, M. Piccin, A. Abbadie, F. Lallement, N. Daval, E. Guiot, A. Rigny, B. Ghyselen, K. Bourdelle, F. Boulanger, S. Cristoloveanu, T. Billon, O. Faynot, C. Deguet, and L. Clavelier, ECS Trans. 25, 351 (2009).

${ }^{27}$ J. Widiez, J.-M. Hartmann, F. Mazen, S. Sollier, C. Veytizou, Y. Bogumilowicz, E. Augendre, M. Martin, F. Gonzatti, M.-C. Roure, J. Duvernay, V. Loup, C. Euvrard-Colnat, A. Seignard, T. Baron, R. Cipro, F. Bassani, A.-M. Papon, C. Guedj, I. Huyet, M. Rivoire, P. Besson, C. Figuet, W. Schwarzenbach, D. Delprat, and T. Signamarcheix, ECS Trans. 64, 35 (2014).

${ }^{28}$ S. Tardif, A. Gassenq, K. Guilloy, N. Pauc, I. Duchemin, G. O. Dias, D. Rouchon, J. M. Hartmann, J. Widiez, J. Escalante, Y. M. Niquet, R. Geiger, T. Zabel, H. Sigg, J. Faist, A. Chelnokov, V. Reboud, V. Calvo, and F. Rieutord, e-print arXiv:1603.06370v1.

${ }^{29}$ P. H. Tan, K. Brunner, D. Bougeard, and G. Abstreiter, Phys. Rev. B 68, 125302 (2003).

${ }^{30}$ M. Mermoux, A. Crisci, F. Baillet, V. Destefanis, D. Rouchon, A. M. Papon, and J. M. Hartmann, J. Appl. Phys. 107, 013512 (2010).

${ }^{31}$ E. Palik, Handbook of Optical Constants of Solids (Academic Press, 1985).

${ }^{32}$ M. Süess, R. Minamisawa, R. Geiger, K. Bourdelle, H. Sigg, and R. Spolenak, Nano Lett. 14, 1249 (2014).

${ }^{33}$ D. Nam, D. S. Sukhdeo, J.-H. Kang, J. Petykiewicz, J. H. Lee, W. S. Jung, J. Vuckovic, M. L. Brongersma, and K. C. Saraswat, Nano Lett. 13, 3118 (2013).

${ }^{34}$ J. E. Proctor, E. Gregoryanz, K. S. Novoselov, M. Lotya, J. N. Coleman, and M. P. Halsall, Phys. Rev. B: Condens. Matter Mater. Phys. 80, 073408 (2009).

${ }^{35}$ B. A. Weinstein and R. Zallen, "Light scattering in solids IV," in Topics in Applied Physics (Springer Berlin Heidelberg, 1984), Vol. 54, Chap. 8, pp. 463-527.

${ }^{36}$ L. D. Yao, F. F. Wang, X. Shen, S. J. You, L. X. Yang, S. Jiang, Y. C. Li, K. Zhu, Y. L. Liu, A. L. Pan, B. S. Zou, J. Liu, C. Q. Jin, and R. C. Yu, J. Alloys Compd. 480, 798 (2009).

${ }^{37}$ A. V. Sapelkin, V. A. Karavanskii, G. Kartopu, M. Es-Souni, and Z. Luklinska, Phys. Status Solidi B 244, 1376 (2007).

${ }^{38}$ B. A. Weinstein and R. Zallen, in Semiconductors and Semimetals, edited by T. Suski and W. Paul (Academic Press, 1998), Vol. 55, p. 151.

${ }^{39}$ E. Scalise, M. Houssa, G. Pourtois, V. V. Afanasev, and A. Stesmans, Phys. E 56, 416 (2014).

${ }^{40}$ O. Robach, J. S. Micha, O. Ulrich, O. Geaymond, O. Sicardy, J. Härtwig, and F. Rieutord, Acta Crystallogr., Sect. A: Found. Crystallogr. 69, 164 (2013).

${ }^{41}$ See http://www.esrf.eu/home/UsersAndScience/Experiments/CRG/BM32/ lauetools-laue-X-ray-microdiffractionanalysis-software.htm for LaueTools: Laue X-Ray Microdiffraction Analysis Software (2010).

${ }^{42}$ J. J. Wortman and R. A. Evans, J. Appl. Phys. 36, 153 (1965).

${ }^{43}$ I. De Wolf, Semicond. Sci. Technol. 11, 139 (1996).

${ }^{44}$ Y. Huo, H. Lin, R. Chen, M. Makarova, Y. Rong, M. Li, T. I. Kamins, J. Vuckovic, and J. S. Harris, Appl. Phys. Lett. 98, 011111 (2011).

${ }^{45}$ D. Rouchon, M. Mermoux, F. Bertin, and J. M. Hartmann, J. Cryst. Growth 392, 66 (2014).

${ }^{46}$ F. Ureña, S. H. Olsen, and J. P. Raskin, J. Appl. Phys. 114, 144507 (2013).

${ }^{47}$ X. Gonze, B. Amadon, P.-M. Anglade, J.-M. Beuken, F. Bottin, P. Boulanger, F. Bruneval, D. Caliste, R. Caracas, M. Cote, T. Deutsch, L. Genovese, P. Ghosez, M. Giantomassi, S. Goedecker, D. R. Hamann, P. Hermet, F. Jollet, G. Jomard, S. Leroux, M. Mancini, S. Mazevet, M. J. T. Oliveira, G. Onida, Y. Pouillon, T. Rangel, G.-M. Rignanese, D. Sangalli, R. Shaltaf, M. Torrent, M. J. Verstraete, G. Zerah, and J. W. Zwanziger, Comput. Phys. Commun. 180, 2582 (2009).

${ }^{48}$ M. Fuchs and M. Scheffler, Comput. Phys. Commun 119, 67 (1999).

${ }^{49}$ X. Gonze, G. M. Rignanese, M. Verstraete, J. M. Beuken, Y. Pouillon, R. Caracas, F. Jollet, M. Torrent, G. Zerah, M. Mikami, P. Ghosez, M. Veithen, J. Y. Raty, V. Olevano, F. Bruneval, L. Reining, R. Godby, G. Onida, D. R. Hamann, and D. C. Allan, Z. Kristallogr. 220, 558 (2005).

${ }^{50}$ D. Olego and M. Cardona, Phys. Rev. B 25, 1151 (1982).

${ }^{51}$ J. B. Renucci, M. A. Renucci, and M. Cardona, Solid State Commun. 9, 1651 (1971). 